\title{
The naming profile in Alzheimer patients parallels that of elderly controls
}

\author{
Tim M. Gale, ${ }^{1,2,3}$ Karen Irvine, ${ }^{1}$ Keith R. Laws, ${ }^{1}$ and Sue Ferrissey ${ }^{2}$ \\ ${ }^{1}$ School of Psychology, University of Hertfordshire, Hatfield, UK \\ ${ }^{2}$ Department of Psychiatry, QEII Hospital, Welwyn Garden City, UK \\ ${ }^{3}$ School of Computer Science, University of Hertfordshire, Hatfield, UK
}

Controversy exists as to whether semantic disruption in Alzheimer's disease (AD) systematically impairs the naming of living things. Moreover, little is known about performance across more specific subcategories. We investigated picture naming in $28 \mathrm{AD}$ patients and 24 controls. To deal with nonnormal distributions, we created 1,000 boot-

10 strap hierarchical regressions and determined which variables (the "nuisance" variables familiarity, word frequency, age of acquisition and visual complexity; category; and control naming) best predicted AD patient naming. Nuisance variables combined, control naming, and category uniquely accounted for 39\%, 36\%, and 3\% of patient naming variance, respectively. Finally, analysis of the AD naming profile across the 10 subcategories mirrored that of controls. Taken together, these findings indicate that while AD naming is, of course, quantita-

15 tively worse than that of controls, it does not qualitatively differ - that is, it is an exaggerated normal profile.

Keywords: Category specific; Alzheimer's disease; Bootstrap; Picture naming; Control; Superordinate.

\section{INTRODUCTION}

Object naming in patients with Alzheimer's disease (AD) is impaired relative to age-matched healthy Q120 elderly controls (e.g., Chertkow \& Bub; 1992; Laws, Gale, Leeson, \& Crawford, 2005); and the types of naming error made by AD patients (e.g., overextending the names of within-category associate items and producing the superordinate, rather

25 than basic or subordinate level name for an item) are widely believed to reflect progressive deterioration in semantic memory function (Chertkow \&

Q1 Bub, 1992; Done \& Gale, 1997). Semantic memory impairment is an early marker of $\mathrm{AD}$, being detect-

30 able even in mild cognitive impairment cases - that is, in pre-AD neuropathology (Adlam, Bozeat, Arnold, Watson, \& Hodges, 2006; Garrard et al., 2001; Vogel, Gade, Stokolm, \& Waldemar, 2005).

There has been increasing interest in recent 35 years as to whether AD systematically inflicts a category-specific impairment in semantic memory. In line with the broader literature on categoryspecific deficits, most studies in AD have focused on the relative impairment of living over nonliving categories. In a recent meta-analysis of 21 picturenaming studies involving 557 AD patients and 509 healthy controls, Laws, Adlington, Gale, MorenoMartínez, and Sartori (2007) found AD patients to be impaired at naming items from both living and nonliving categories. Although more studies revealed deficits for living than nonliving things (13:8), no significant difference emerged between the effect sizes for living and nonliving things. Minimally, this casts a certain amount of doubt on the notion that $\mathrm{AD}$ patients suffer from a relative impairment in naming living things, although the question remains as to why some studies report category effects in $\mathrm{AD}$, whereas others do not.

One problem with comparing previous picturenaming studies in $\mathrm{AD}$ is the considerable variability

The authors are grateful to Peter Simmons, Hema Ananth, and all participants in the study. We also acknowledge the contributions of three reviewers.

Address correspondence to Tim M. Gale, Department of Psychiatry, QEII Hospital, Howlands, Welwyn Garden City, Hertfordshire, AL7 4HQ, UK (E-mail: t.gale@herts.ac.uk).

(C) 2008 Psychology Press, an imprint of the Taylor \& Francis Group, an Informa business 
in the number and types of living and nonliving stimuli used by different researchers. For example, Laws et al. (2007) report that studies have used a wide range of 20-120 items. Although Laws et al.

65 (2007) found that the number of stimulus items did not significantly predict effect sizes for either living or nonliving things, the emergence of category effects may still depend crucially on the specific choice of items used. In this context, the question

70 of whether living or nonliving impairments reflect impoverished naming across the majority of subcategories within either domain (living or nonliving), or only a small subset, has not been systematically investigated in previous studies. The

75 number of living and nonliving subcategories, as well as the specific choice of items representing each subcategory, may therefore influence the presence and direction of emergent category effects (Aronoff et al., 2006).

80 Tippett, Grossman, and Farah (1996) showed that the emergence of a group category effect in AD patients was contingent on whether or not stimuli were matched across living and nonliving things on so-called "nuisance variables" (e.g.,

85 familiarity, visual complexity, word-frequency, and so on). Subsequently, recent studies of category specificity in $\mathrm{AD}$ have taken great care to match living and nonliving stimuli on as many relevant nuisance variables as possible (for a fuller

90 exposition of the degree of variable matching, see Moreno-Martínez \& Laws, 2007). Given the level of discord between living and nonliving things on nearly all such variables (Barbarotto, Capitani, \& Laiacona, 2001; Gale \& Laws, 2006), the choice of

95 items available for inclusion in matched sets of pictures is reduced, especially when stimuli are drawn from a single source such as the Snodgrass and Vanderwart (1980) corpus. For example, living things in the Snodgrass and Vanderwart cor-

100 pus are typically less familiar and have lower word frequency and higher visual complexity than nonliving things, and so there is an inherent bias, when matching between living and nonliving domains, to exclude living-thing items that might

105 exaggerate this tendency (and to include less familiar nonliving things that counter the bias). Consequently, some items and subcategories are more widely represented in studies of AD naming than others. A useful approach when comparing

110 living and nonliving things may therefore be to match within each domain for the number of different subcategories (fruits, vegetables, clothing, tools, etc.) and also the number of items representing each subcategory. A more detailed analysis of

115 AD naming error profiles across a range of subcategories within living and nonliving domains is also required for a more specific test of some models of category specificity.

Additionally, the relative level of naming accuracy in healthy control groups, with respect to patients, can strongly affect the profile of patient impairment when the two groups are compared statistically. A series of experiments examining category specific naming in AD by Laws et al. (2005) showed that the emergence of a category 125 effect and, perhaps more importantly, the direction of the effect, was modulated by the overall performance of the control group. Most healthy control subjects perform close to ceiling level in standard object-naming tasks, and this is espe- 130 cially so for studies that have presented stimuli from the Snodgrass and Vanderwart (1980) corpus (see Laws et al., 2007). Indeed, the vast majority of studies examining picture naming in $\mathrm{AD}$, and other neuropathologies, have selected their stimuli 135 from this corpus (see Laws, 2005, for a discussion). Ceiling level performance of control participants invalidates some important assumptions of statistical tests that compare control and patient group variances (Laws, 2005; Laws et al., 2005; 140 Laws, Leeson, \& Gale, 2003). Methods of data analysis that do not succumb to problems with nonnormal distributions are therefore essential, and bootstrap methods comprise one such alternative set of approaches for dealing with such data 145 (e.g., Moreno-Martínez \& Laws, 2007). These methods, which require far fewer assumptions than standard parametric tests, are suitable in circumstances where many zero data points exist in the dataset (e.g., controls who score very highly, 150 or patients who perform at, or near, floor level). With bootstrap techniques, a relevant test statistic $(t, F, r$, etc.) is selected, and this statistic is then computed for $n$ bootstrap samples - that is, $n$ permutations of the original group data. When this occurs with replacement, each data point returns to the sampling pool and may be redrawn numerous times. After many permutations, this results in a distribution of test statistics (rather than data points), which can be analyzed. Hence bootstrap 160 methods may be applied to data that have been collected using traditional, easy-to-name, stimuli, even when ceiling effects are present (Delucchi \& Bostrom, 2004).

In this study, we compared the object naming 165 profiles of 28 probable AD patients and 24 healthy elderly control participants. We used a set of 100 pictures drawn from the Snodgrass and Vanderwart (1980) corpus, which was specifically selected to control for the number of living and nonliving 170 subcategories (i.e., animals, birds, clothing, furniture, etc.) and also the number of items representing 
each of those subcategories. The pictures are matched across domain (living vs. nonliving) for 175 familiarity, word frequency, and visual complexity. We used bootstrap hierarchical regression analyses to establish the best predictors for the AD naming profile, and we also examined the naming profile of patients and controls across subcategories.

\section{METHOD}

\section{Materials}

A total of 100 pictures depicting items from 10 different subcategories were selected from the Snodgrass and Vanderwart (1980) corpus. We used

185 the grayscale versions of these stimuli that have been created by Rossion and Pourtois (2004), and which contain greater amounts of surface texture than the original line drawings. Items were selected from 5 living-thing subcategories (animals, birds,

190 body parts, fruit, and vegetables) and 5 nonliving subcategories (clothing, furniture, musical items, tools, and vehicles), with 10 different items representing each subcategory. The pictures were presented on laminated cards of approximately 10

$195 \mathrm{~cm}^{2}$. Living and nonliving things were matched for: concept familiarity $(3.24 \pm 1.01$ vs. $3.53 \pm 0.87)$, $F(1,98)=2.41, p>.1$; visual complexity $(3.01 \pm$ 0.93 vs. $3.03 \pm 0.85), F(1,98)<1, p>.9$; and $\log$ word frequency $(1.11 \pm 0.64$ vs. $1.13 \pm 0.75), F(1$,

$20098)<1, p=.88$; from Kuçera \& Francis, 1967), but not for age of acquisition $(3.6 \pm 1.04$ vs. $3.41 \pm$ $1.18), F(1,98)=4.29, p=.04$. This set of stimuli is also reported in Gale and Laws (2006), and Gale, Laws, and Foley (2006), and a list of all items 205 appears in the Appendix.

\section{Participants}

\section{Patients}

A total of 28 patients with probable AD were recruited from a consecutive series of attendees at

210 an outpatients' memory clinic in the United Kingdom. All participants had been assessed for probable AD using National Institute of Clinical Effectiveness (NICE) criteria for diagnosis of AD (NICE, 2007) which includes elimination of other

215 possible pathologies by means of detailed assessment of history/onset, detailed neuropsychological assessment, and, in some cases, neuroimaging. Any patients who were judged by their treating consultant not to have capacity to give informed consent

220 were excluded. All included patients had normal, or corrected-to-normal, vision, and all spoke English as their first language. The AD group comprised 9 males and 19 females, and mean age was 83.3 years $(S D=6.9$; range $=71-98$ years $)$. The average Mini Mental State Examination (MMSE: Folstein, Folstein, \& McHugh, 1975) score was 22.1 ( $S D=4.5$; range $=14-30)$. One individual with probable AD scored 30 on the MMSE at the time of testing (all others scored less than 28). However, this person presented with marked anomia and had been given 230 a probable diagnosis of AD by her treating clinician, so we included her in the study on this basis. Mean predicted premorbid IQ score for the group (derived from National Adult Reading Test, NART, scores: Nelson, 1982) was 109.4 ( $\pm 7.6 ; 235$ range 95-119).

\section{Controls}

A total of 24 elderly control participants (13 male, 11 female) of mean age $78(S D=6)$ years were recruited. Although controls and AD patients 240 were not matched exactly on age, this factor was accounted for in later analyses. The controls were recruited through their general practitioner, who had screened them for good health. All were healthy, had no history of cognitive impairment, 245 psychiatric illness, any form of brain injury, or alcohol or drug abuse. All had normal, or corrected-to-normal, eyesight, and all spoke English as their first language.

\section{Procedure}

The study was ethically approved by the National Health Service (NHS) Hertfordshire Research Ethics Committee. The majority of participants (patients and controls) completed the naming task in their own homes, seated comfortably at a table. 255 The picture cards were presented, one at a time, in a pseudorandomly determined order, and the participant was asked to name each item in turn. The exact response was recorded verbatim on a response sheet for later scoring. The pictures were 260 presented in two blocks, each of 50 cards, with a short break between blocks.

Where a picture could legitimately be referred to by more than one name (e.g., sailboat/yacht, chicken/hen, sofa/couch, lorry/truck, etc.), the 265 alternative names were accepted as correct. Similarly, when presented in such a stylized format, some items were difficult to distinguish from visually similar associates, (e.g., violin/viola, cabbage/ lettuce). In such cases, either name was accepted as 270 correct. Finally, items that were named at a more specific level (e.g., "overcoat" for coat; "trilby" for hat) were accepted as correct provided that the 
subordinate level name given was appropriate to

275 the specific depiction of the item. Responses were scored by two raters (T.G. and K.I.), and a consensus was reached by discussion for any items where the raters had disagreed.

\section{RESULTS}

\section{Living versus nonliving}

AD patients named significantly more nonliving (mean $=81.3 \%, S D=19.3 \%)$ than living items $($ mean $=71.6 \%, S D=25.6 \%), F(1,98)=4.59, p=$ .035. Controls named slightly more nonliving $285($ mean $=96 \%, S D=9.4 \%)$ than living items (mean $=95 \%, S D=10 \%$ ) but the difference did not reach significance, $F(1,98)<1$.

We computed skewness and kurtosis statistics $\left(g_{1}\right.$ and $g_{2}$ ) for both the patient and the healthy 290 control data. For patients, skewness was -1.17 , and kurtosis was 0.73. D'Agostino, Belanger, and D'Agostino's (1990) test for skewness failed to reject the null hypothesis that the distribution was symmetrical: $z g_{1}=-4.2$. Further, the D'Agostino-

295 Pearson omnibus test for normality, which uses both $g_{1}$ and $g_{2}$ as input, revealed that the distribution did differ significantly from normality: $K^{2}=$ $19.8, p<.0001$. For the controls, skewness was 3.10, and kurtosis was 10.81. D'Agostino et al.'s

300 test for skewness failed to reject the null hypothesis that the distribution was symmetrical: $z g_{1}=-7.6$. Further, the D'Agostino-Pearson omnibus test for normality revealed that the distribution differed significantly from normality: $K^{2}=88.1, p<.0001$.

305 Given that all the variables we examined correlated with patient naming (Table 1), we used a bootstrap multiple regression to estimate the degree of variance in the $\mathrm{AD}$ patient naming data that was explained by each predictor (familiarity,

310 word frequency, age of acquisition, visual complexity, category - living vs. nonliving - and control naming performance). We created 1,000 bootstrap samples, each equal in size to the original sample and each using random, with-replacement, sampling. One single bootstrap sample 315 might therefore contain multiple instances of a single data point and no instances of a different data point. Multiple hierarchical regressions were run for each of the 1,000 bootstrap samples to determine the contribution of each predictor in explaining 320 the outcome variance within each model. Table 2 shows the contribution of each predictor variable in accounting for naming in the AD patients.

Each hierarchical regression analysis included three blocks of predictors. In Model 1, we entered 325 the so-called "nuisance variables" (familiarity, word frequency, age of acquisition, and visual complexity) together in Block 1, followed by "category" in Block 2, and finally "control performance" in Block 3 (Table 2, Model 1). This revealed 330 that the nuisance variables accounted for $39 \%$ of the variance in patient naming. Category (living vs. nonliving) was also significant, accounting for $10 \%$ of variance after controlling for the nuisance variables. Finally, control performance was 335 highly significant and accounted for almost $30 \%$ of the remaining variance after controlling both for the effects of nuisance variables and for the effects of category. Finally, the 1,000 bootstrapped hierarchical regression analyses were 340 rerun, but this time changing the order of steps to: "nuisance variables," "control performance," and finally "category" to determine the amount of variance attributable to category after controlling for nuisance variables and the naming difficulty index, as measured by control naming performance (Table 2, Model 2). Here category accounted for only a small (3\%), though significant, amount of patient naming variance after controlling for all of the nuisance variables and the difficulty 350 index for controls $\left(R^{2}\right.$ change $\left.=.36, p<.0001\right)$. In combination, these hierarchical regression analyses revealed that category and control difficulty accounted, respectively, for $3-10 \%$ and $29-36 \%$ of the variance in $\mathrm{AD}$ naming.

TABLE 1

Correlations between $A D$ naming and nuisance variables

\begin{tabular}{|c|c|c|c|c|c|c|}
\hline & $V C$ & $A A$ & $W F$ & Control naming & Category & AD naming \\
\hline Familiarity & $-.40^{* * *}$ & $-.69 * * *$ & $.40 * * *$ & $.30^{* *}$ & .16 & $.51 * * *$ \\
\hline Visual comp & - & $.39 * * *$ & -.16 & -.11 & .01 & $-.22 *$ \\
\hline AA & & - & $-.52 * * *$ & $-.43 * * *$ & $.22 *$ & $-.59 * * *$ \\
\hline WF $(\log )$ & & & - & .17 & .01 & $.38 * * *$ \\
\hline Cont. Naming & & & & - & .10 & $.79 * * *$ \\
\hline Category & & & & & - & $.21 *$ \\
\hline
\end{tabular}

Note. $\mathrm{AD}=$ Alzheimer's disease $\mathrm{VC}=$ visual complexity. $\mathrm{AA}=$ age of acquisition. $\mathrm{WF}=$ word frequency. Probability two-tails: ${ }^{*} p<.05 .{ }^{* *} p<.01 .{ }^{* * *} p<.001$. 
TABLE 2

Results of 1,000 bootstrap regression analyses using two different hierarchical models

\begin{tabular}{|c|c|c|c|c|c|}
\hline Model & Step & Variable & $R^{2}$ & $\Delta R^{2}$ & $p$ \\
\hline \multirow[t]{4}{*}{1} & 1 & $\begin{array}{l}\text { Familiarity, visual } \\
\text { complexity, word fre- } \\
\text { quency, and AoA }\end{array}$ & .39 & .39 & \\
\hline & 2 & Category & .49 & .10 & .0052 \\
\hline & 3 & Control naming & .78 & .29 & $<.0001$ \\
\hline & & $\begin{array}{r}\text { Overall model } \\
\text { adjusted } R^{2}\end{array}$ & .78 & & \\
\hline \multirow[t]{3}{*}{2} & 1 & $\begin{array}{l}\text { Familiarity, visual } \\
\text { complexity, word fre- } \\
\text { quency, and AoA }\end{array}$ & .39 & .39 & \\
\hline & 2 & Control naming & .75 & .36 & $<.0001$ \\
\hline & 3 & Category & .78 & .03 & .02 \\
\hline
\end{tabular}

Note. AoA $=$ age of acquisition.

\section{More closely matched samples}

Recent work shows that the sex of participants may interact with semantic category, both for patients and for healthy participants. In particular,

360 men show better performance with some nonliving subcategories, while women show an advantage with some living subcategories (for reviews, see Gainotti, 2005; Laiacona, Barbarotto, \& Capitani, 2006). Because our groups were not closely

365 matched for sex ratio, we reran the analyses on a subset of patients and controls who were closely matched: ${ }^{1} 18$ AD patients ( 9 male, 9 female) with a mean age 79.6 years and 22 elderly controls (11 male, 11 female) with a mean age 79 years. We also

370 removed the one AD patient with a MMSE score of 30 . The bootstrap analyses did not differ from those described above for the full sample (see Table 3).

\section{Subcategories}

375 The profiles of subcategory naming for AD patients and controls are displayed in Figure 1. Body parts were named most accurately by patients and controls, a pattern that is not typical of the overall living-thing naming profile. Similarly, the

380 naming of musical instruments was more consistent with the naming accuracy levels observed in several of the living-thing subcategories.

The range of item accuracies for AD patients was $10.7 \%$ to $100 \%$. The least accurately named 385 (all below 40\%) were: artichoke, pepper, pumpkin,

\footnotetext{
${ }^{1}$ Unfortunately information about length of education was not available for patients or controls.
}

TABLE 3

Results of 1,000 bootstrap regression analyses on a subset of closely matched patient and control samples using two different hierarchical models

\begin{tabular}{|c|c|c|c|c|c|}
\hline Model & Step & Variable & $R^{2}$ & $\Delta R^{2}$ & $p$ \\
\hline \multirow[t]{4}{*}{1} & 1 & $\begin{array}{l}\text { Familiarity, visual } \\
\text { complexity, word } \\
\text { frequency, and } \\
\text { AoA }\end{array}$ & .33 & .33 & \\
\hline & 2 & Category & .45 & .11 & .004 \\
\hline & 3 & Control naming & .74 & .30 & $<.0001$ \\
\hline & & $\begin{array}{l}\text { Overall model } \\
\text { adjusted } R^{2}\end{array}$ & .78 & & \\
\hline \multirow[t]{3}{*}{2} & 1 & $\begin{array}{l}\text { Familiarity, visual } \\
\text { complexity, word } \\
\text { frequency, and } \\
\text { AoA }\end{array}$ & .33 & .33 & \\
\hline & 2 & Control naming & .70 & .37 & $<.0001$ \\
\hline & 3 & Category & .74 & .04 & .02 \\
\hline
\end{tabular}

Note. $\mathrm{AoA}=$ age of acquisition.

French horn, cherry, eagle, peach, guitar, ostrich, and asparagus (notably, all living things or musical items). The most accurately named (all at $100 \%$ ) were: banana, bike, bird, bed, car, hammer, foot, hat, trousers, lips, dog, chair, shoe, scissors, 390 and ear.

After $Z$-transforming the within-group naming performance for $\mathrm{AD}$ patients and for elderly controls, a remarkably similar profile of naming emerged in the two groups (Figure 2). This suggests that the levels of difficulty shown by controls are exaggerated in AD patients and that this pattern emerges consistently across all subcategories. For example, both groups clearly found vegetables and musical instruments the most difficult to name 400 subcategories; and both found body parts and clothing the easiest to name.

\section{DISCUSSION}

This study examined two issues that may underpin inconsistent findings in the study of category spe- 405 cific semantic impairments in AD. First, we examined the influence of the so called "nuisance variables," which are known to differ across living and nonliving domains. Second, and more importantly, we examined the treatment of control data 410 and the associated problem of ceiling effects. We proposed the use of bootstrap analyses as a solution: As noted, bootstrap techniques are one way to circumvent the problems associated with nonnormal distributions, which often emerge when 415 contrasting neurologically impaired and unimpaired groups (Delucchi \& Bostrom, 2004). 

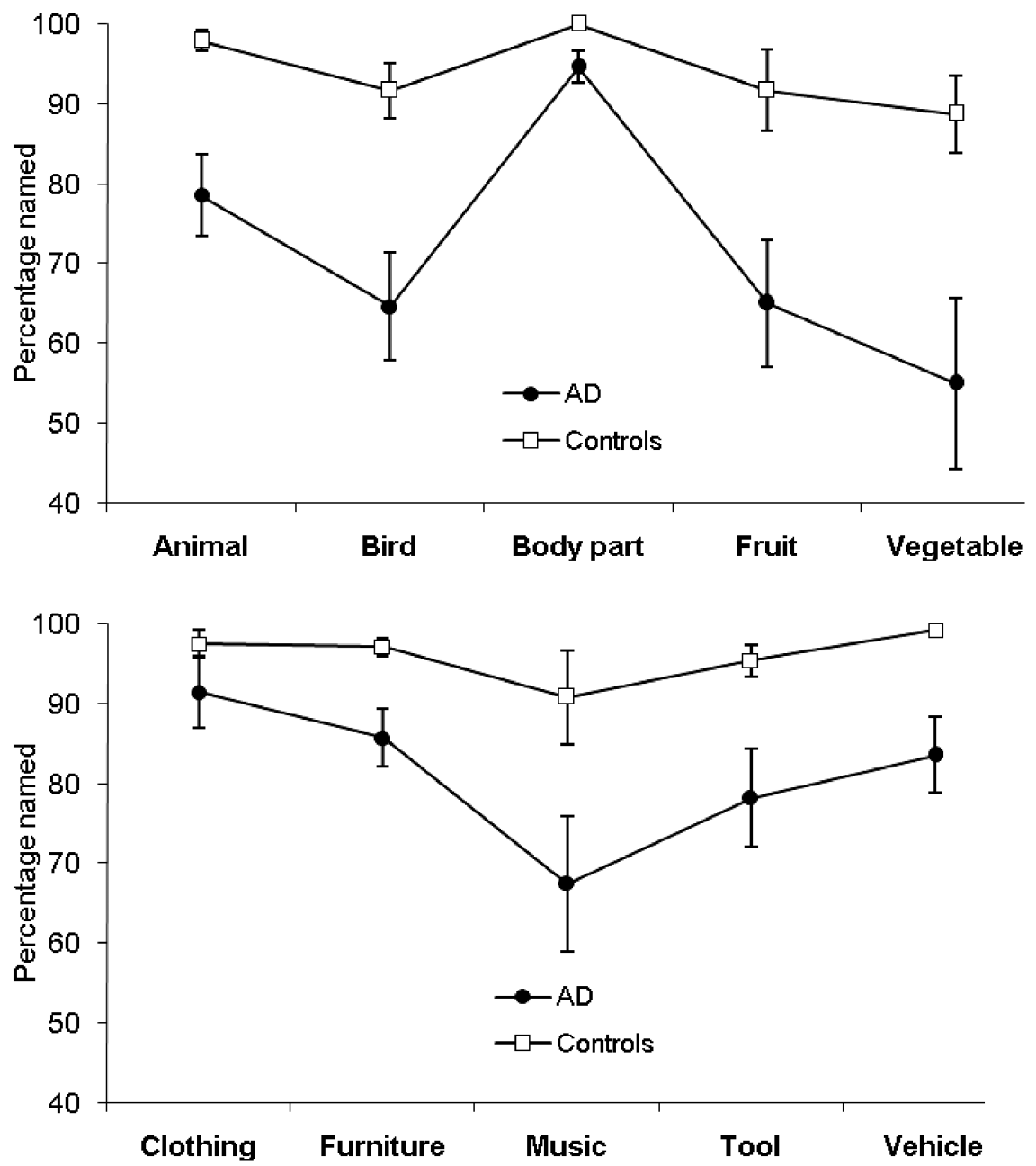

Figure 1. Mean naming (percentage) for Alzheimer's disease (AD) patients and elderly healthy controls in five living and five nonliving subcategories. Bars $=$ standard errors.

Many studies have now investigated categoryspecific naming performance in AD using rigor-

420 ously controlled stimuli; however, their findings are not wholly consistent (for a review, see Laws et al., 2007). Although most reports of categoryspecific impairments in AD patients record livingthing impairments (e.g., Grossman, Robinson,

425 Biassou, White-Devine, \& D'Esposito, 1998; Mauri, Daum, Sartori, Riesch, \& Birbaumer, 1994; Silveri, Daniele, Giustolisi, \& Gainotti, 1991), others describe both living and nonliving deficits within the same group of patients (Gonnerman,

430 Andersen, Devlin, Kempler, \& Seidenberg, 1997; Laws et al., 2005; Laws et al., 2003; MorenoMartínez, Tallón-Barranco, \& Frank-García, 2007; Tippett et al., 1996; Zannino, Perri, Carlesimo, Pasqualetti, \& Caltagirone, 2002). Furthermore,

435 Laws et al's (2007) meta-analysis of categoryspecific picture naming in AD patients highlighted the fact that while more studies have reported significant category effects for living things, the effect sizes for living and nonliving things did not significantly differ. The greater number of previous stud- 440 ies reporting living-thing impairments has, perhaps, encouraged the impression that $\mathrm{AD}$ patients show a differential living-thing category disadvantage. In concurrence with most previous studies, we found that AD patients named signifi- 445 cantly fewer living than nonliving things and, furthermore, that this could not be readily attributed to any differences in nuisance variables (at least those that were matched statistically across living and nonliving domains).

As with many similar studies of picture naming in AD that have used the Snodgrass and Vanderwart (1980) images, the data from our healthy controls were at ceiling and may have therefore masked any "normal" category effect-especially 455 since the patient data were also nonnormally distributed. What is almost certain is that any conventional statistical comparison of the $\mathrm{AD}$ and control groups may well have led to an unreliable conclusion 

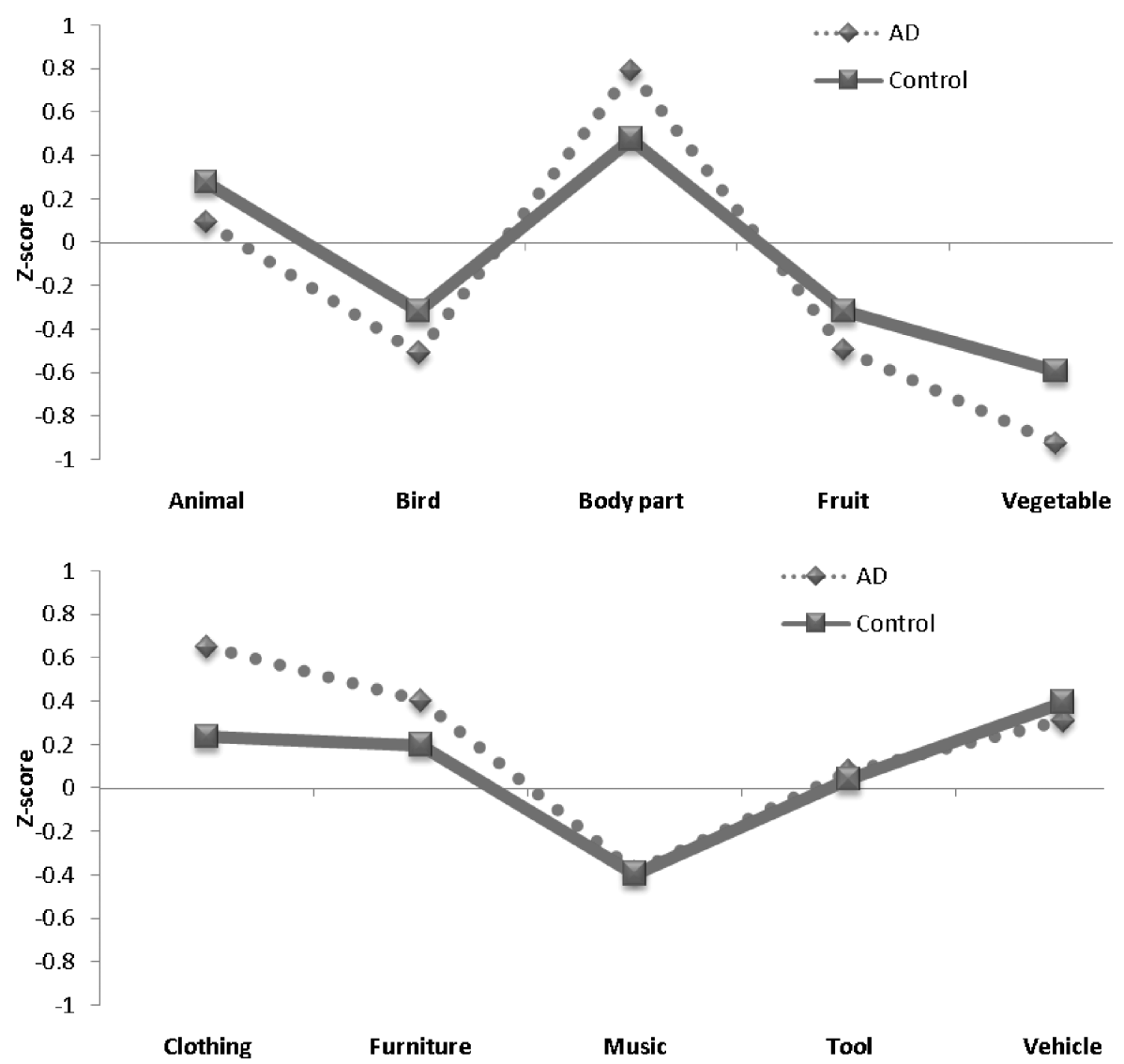

Figure 2. Standardized Z-profiles for Alzheimer's disease (AD) patients and elderly healthy controls across five living and five nonliving subcategories.

460 regarding the size and direction of category effects in these patients (see Laws, 2005; Laws et al., 2005). As we have argued, bootstrap analyses address some of the problems associated with heavily skewed distributions, and, in the current study, we

465 used bootstrap hierarchical regression analyses to determine the specific roles played by three types of variable (nuisance variables per se, a control group difficulty index, and category). As already noted, the use of unmatched stimuli has been high-

470 lighted as one possible reason why patients may show poorer performance with living than nonliving things in some previous studies (Tippett et al., 1996). Although we matched items across category (living vs. nonliving) on some nuisance variables

475 (familiarity, visual complexity, log word frequency), these variables, combined with age of acquisition, still accounted for a large proportion of the variance in patient naming $(39 \%)$.

The persistence of a significant, albeit small, cat-

480 egory effect, even after controlling for nuisance variables, does not alone confirm that the category effect is a consequence of the neurological damage. Rather, it is also vital to establish whether the category effect is larger than that which might be expected in healthy controls. A large amount of 485 variance in patient naming was uniquely explained by the difficulty index derived from elderly healthy controls (approximately 29\%). By contrast, although category did significantly predict patient naming, it uniquely accounted for just 3\% of the 490 variance after controlling for the other variables (i.e., 10 times less than the control difficulty index). While we would not argue that the direction and size of this difference in controls would invariably occur across different stimulus sets, the level and 495 direction of difficulty that exists for healthy controls must be established on any specific stimulus set being used.

Both the regression analyses and, furthermore, the profile across subcategories indicate that 500 despite the obvious quantitative difference between patient and control performance, they do not differ qualitatively. As far as we are aware, no previous study has examined such a broad range of categories in $\mathrm{AD}$ patients, at least with respect to picture 505 naming. Critically, the AD patients and healthy controls showed similar difficulty profiles across the five living and the five nonliving categories. For example, although AD patients show greater difficulty with 
510 naming vegetables, this was also the most difficult category for controls to name. Notably both groups show substantial naming variability across subcategories, and this again underscores the importance of stimulus choice when examining cat-

515 egory effects. As with previous studies (Barbarotto et al., 2001; Gale \& Laws 2006; Gale et al., 2006; Laws, Gale, Frank, \& Davey, 2002a), body parts and musical instruments appear to be atypically good and poor when referenced to living and non-

520 living categories, respectively, both for AD patients and for controls. In other words, naming in AD patients reflects a similar pattern of task difficulty expressed by healthy elderly controls.

Our findings are consistent with the patient per-

525 formance being an exaggeration of normal healthy control performance (see Moreno-Martínez \& Laws, 2007, in press; Perri et al., 2003). The presence of a considerable category effect in neurologically normal participants may well have been

530 "hidden" by ceiling effects in the control data of previous studies. Indeed, the presence of a normal category advantage (whether living or nonliving) accords with recent findings in healthy participants (Brousseau \& Buchanan, 2004; Coppens \&

535 Frisinger, 2005; Filliter, McMullen, \& Westwood, 2004; Låg, 2005; Låg, Hveem, Ruud, \& Laeng, 2006; Laws, 1999, 2000; Laws \& Hunter, 2006; Laws \& Neve, 1999; Laws, Leeson, \& Gale, 2002b; Lloyd-Jones \& Luckhurst, 2002; McKenna \&

540 Parry, 1994). With the recent accumulation of studies documenting category effects in healthy participants, it is pertinent to ask whether, and indeed how, extant models of category specificity incorporate the notion of category effects in the 545 healthy brain.

Current models of category specificity have been designed to specifically account for patient deficits rather than to make predictions about normal category effects, and so may not make obvious predic-

550 tions about category effects in normal cognition. The closest to a normal model is, indeed, the artifactual (nuisance variable) account, and this would typically predict a nonliving advantage, though not of course for matched stimuli. Although the

555 "domain-specific" account (Caramazza \& Shelton, 1998) does not make specific predictions about normal category biases, we might expect the preferential processing of those categories that have dedicated domains (e.g., foodstuffs, animals,

560 tools) in neurologically unimpaired individuals (see Laws, 2000). Our data provide no evidence of differential impairment in any subcategory of living or nonliving domains. This suggests that the naming of $\mathrm{AD}$ patients reflects the same pattern of task

565 difficulty as that seen in healthy elderly controls - that is, there are no qualitative differences attributable to the disease process itself. Rather, at least within the context of AD, the disease process affects categories in an additive manner, rather than selectively affecting specific neural subsystems of 570 knowledge. Whatever predictions may or may not be derived from extant models of category specificity, this study underlines the importance of examining the performance of neurologically healthy participants when documenting category-specific 575 naming deficits in neurological patients, and the need for models of category specificity to address the finding of normal category effects.

Original manuscript received 11 April 2008 Revised manuscript accepted 22 July 2007 First published online

\section{REFERENCES}

Adlam, A.-L. R., Bozeat, S., Arnold, R., Watson, P., \& Hodges, J. R. (2006). Semantic knowledge in mild cognitive impairment and mild Alzheimer's disease. Cortex, 42, 675-684.

Aronoff, J. M., Gonnerman, L. M., Almor, A., Arunachalam, S., Kempler, D., \& Andersen, E. S. (2006). Information content versus relational knowledge: Semantic deficits in patients with Alzheimer's disease. Neuropsychologia, 44, 21-35.

Barbarotto, R., Capitani, E., \& Laiacona, M. (2001). Living musical instruments and inanimate body parts? Neuropsychologia, 39, 406-414.

Brousseau, G., \& Buchanan, L. (2004). Semantic cate- 595 gory effect and emotional valence in female university students. Brain \& Language, 90, 241-248.

Caramazza, A., \& Shelton, J. R. (1998). Domain-specific knowledge systems in the brain: The animate-inanimate distinction. Journal of Cognitive Neuroscience, 600 $10,1-34$.

Chertkow, H., \& Bub, D. (1990). Semantic memory loss in dementia of Alzheimer's type: What do various measures measure? Brain, 113, 397-417.

Coppens, P., \& Frisinger, D. (2005). Category-specific 605 naming effect in non-brain damaged individuals. Brain \& Language, 94, 61-71.

D’Agostino, R. B., Belanger, A., \& D'Agostino, R. B., Jr. (1990). A suggestion for using powerful and informative tests of normality. The American Statistician, 44, 316-321.

Delucchi, K. L., \& Bostrom, A. (2004). Methods for analysis of skewed data distributions in psychiatric clinical studies: Working with many zero values. American Journal of Psychiatry, 161, 1159-1168.

Done, D. J., \& Gale, T. M. (1997). Attribute verification in dementia of Alzheimer type: Evidence for the preservation of distributed concept knowledge. Cognitive Neuropsychology, 14, 547-571.

Filliter, J. H., McMullen, P. A., \& Westwood, D. (2005). 620 Q6 Manipulability and living/non-living category effects on object identification. Brain \& Cognition, 57, 61-65.

Folstein, M. F., Folstein, S. E., \& McHugh, P. R. (1975). "Mini-mental state": A practical method for grading 
625 the cognitive state of patients for the clinician. Journal of Psychiatric Research, 12, 189-198.

Gainotti, G. (2005). The influence of gender and lesion location on naming disorders for animals, plants and artefacts. Neuropsychologia, 43, 1633-1644.

630 Gale, T. M., \& Laws, K. R. (2006). Category-specificity can emerge from bottom-up visual characteristics: Evidence from a modular neural network. Brain \& Cognition, 61, 269-279.

Gale, T. M., Laws, K. R., \& Foley, K. (2006). Crowded

635 and sparse domains in object recognition: Consequences for categorisation and naming. Brain \& $\mathrm{Cog}$ nition, 60, 139-145.

Garrard, P., Lambon-Ralph, M. A., Watson, P. C., Powis, J., Patterson, K., \& Hodges, J. R. (2001). Lon-

640 gitudinal profiles of semantic impairment for living and nonliving concepts in dementia of Alzheimer's type. Journal of Cognitive Neuroscience, 13, 892-909.

Gonnerman, L. M., Andersen, E. S., Devlin, J. T., Kempler, D., \& Seidenberg, M. S. (1997). Double dissocia-

645 tion of semantic categories in Alzheimer's disease. Brain \& Language, 57, 254-279.

Grossman, M., Robinson, K., Biassou, N., WhiteDevine, T., \& D'Esposito, M. D. (1998). Semantic memory in Alzheimer's disease: Representativeness,

650 ontologic category, and material. Neuropsychology, $12,34-42$.

Kuçera, H., \& Francis, W. (1967). Computational analysis of present-day American English. Providence, RI: Brown University Press.

655 Låg, T. (2005). Category-specific effects in object identification: What is "normal"? Cortex, 41, 833-841.

Låg, T., Hveem, K., Ruud, K. P. E., \& Laeng, B. (2006). The visual basis of category effects in object identification: Evidence from the visual hemi-field paradigm.

660 Brain \& Cognition, 60, 1-10.

Laiacona, M., Barbarotto, R., \& Capitani, E. (2006). Human evolution and the brain representation of semantic knowledge: Is there a role for sex differences. Evolution and Human Behaviour, 27, 158-168.

665 Laws, K. R. (1999). Gender affects naming latencies for living and non-living things: Implications for familiarity. Cortex, 35, 729-733.

Laws, K. R. (2000). Category-specific naming errors in normal subjects: The influence of evolution and expe-

670 rience. Brain \& Language, 75, 123-133.

Laws, K. R. (2005). Illusions of normality: A methodological critique of category-specific naming. Cortex, $41,842-851$.

Laws, K. R., Adlington, R. L., Gale, T. M., Moreno-

675 Martínez, F. J., \& Sartori, G. (2007). A meta-analytic review of category naming in Alzheimer's disease. Neuropsychologia, 45, 2674-2682.

Laws, K. R., Gale, T. M., Frank, R., \& Davey, N. (2002a). Visual similarity is greater for line drawings

680 of nonliving than living things: The importance of musical instruments and body-parts. Brain \& Cognition, 48, 421-424.

Laws, K. R., Gale, T. M., Leeson, V. C., \& Crawford, J. (2005). When is category specific in Alzheimer's dis685 ease? Cortex, 41, 452-463.

Laws, K. R., \& Hunter, M. Z. (2006). The impact of colour, spatial resolution and presentation speed on category naming. Brain \& Cognition, 62, 89-97.

Laws, K. R., Leeson, V. C., \& Gale, T. M. (2002b). The

690 effect of "masking" on picture naming. Cortex, 38, $137-147$.
Laws, K. R., Leeson, V. C., \& Gale, T. M. (2003). Inflated and contradictory category naming deficits in Alzheimer's disease? Brain \& Cognition, 53, 416-418.

Laws, K. R., \& Neve, C. (1999). A "normal” category- 695 specific advantage for naming living things. Neuropsychologia, 37, 1263-1269.

Lloyd-Jones, T. J., \& Luckhurst, L. (2002). Outline shape is a mediator of object recognition that is particularly important for living things. Memory \& Cognition, 30, 489-498.

Mauri, A., Daum, I., Sartori, G., Riesch, G., \& Birbaumer, N. (1994). Category-specific semantic impairment in Alzheimer's disease and temporal lobe dysfunction: A comparative study. Journal of Clinical \& Experimental Neuropsychology, 16, 689-701.

McKenna, P., \& Parry, R. (1994). Category-specificity in the naming of natural and man-made objects: Normative data from adults and children. Neuropsychological Rehabilitation, 4, 255-281.

Moreno-Martínez, F. J., \& Laws, K. R. (2007). An attenuation of the "normal" category effect in patients with Alzheimer's disease: A review and bootstrap analysis. Brain \& Cognition, 63, 167-173.

Moreno-Martínez, F. J., \& Laws, K. R (in press). No 715Q5 category-specificity in Alzheimer's disease: A normal aging effect. Neuropsychology.

Moreno-Martínez, F. J., Tallón-Barranco, A., \& FrankGarcía, A. (2007). Enfermedad de Alzheimer, deterioro categorial y variables relevantes en la denominación de objetos [Alzheimer's disease, categoryspecific impairment and relevant variables in object naming]. Revista de Neurología, 44, 129-133.

Nelson, H. E. (1982). National Adult Reading Test (NART): Test manual. Windsor, UK: NFER Nelson.

NICE. (2007). Alzheimer's disease-donepezil, galantamine, rivastigmine (review) and memantine (NICE Technology Appraisal TA111).

Perri, R., Carlesimo, G. A., Zannino, G. D., Mauri, M., Muolo, B., Pettenati, C., et al. (2003). Intentional and automatic measures of specific-category effect in the semantic impairment of patients with Alzheimer's disease. Neuropsychologia, 41, 1509-1522.

Rossion, B., \& Pourtois, G. (2004). Revisiting Snodgrass and Vanderwart's object pictorial set: The role of surface detail in basic-level object recognition. Perception, 33, 217-236.

Silveri, M. C., Daniele, A., Giustolisi, L., \& Gainotti, G. (1991). Dissociation between knowledge of living and non living things in dementia of the Alzheimer type. Neurology, 41, 545-546.

Snodgrass, J. G., \& Vanderwart, M. (1980). A standardised set of 260 pictures: Norms for name agreement, image agreement, familiarity, and visual complexity. Journal of Experimental Psychology: Human Learning \& Memory, 6, 174-215.

Tippett, L. J., Grossman, M., \& Farah, M. J. (1996). The semantic memory impairment of Alzheimer's disease: Category specific? Cortex, 32, 143-153.

Vogel, A., Gade, A., Stokolm, J., \& Waldemar, G. (2005). Semantic memory impairment in the earliest phases of Alzheimer's disease. Dementia and Geriatric Cognitive Disorders, 19, 75-81.

Zannino, G. D., Perri, R., Carlesimo, G. A., Pasqualetti, P., \& Caltagirone, C. (2002). Category-specific impairment in patients with Alzheimer's disease as a function of disease severity: A cross sectional investigation. Neuropsychologia, 40, 2268-2279. 


\section{APPENDIX}

\section{LIST OF STIMULI USED}

Animals: bear, cow, dog, elephant, giraffe, goat, horse, lion, sheep, squirrel

Birds: bird, chicken, duck, eagle, ostrich, owl, peacock, penguin, rooster, swan

765 Body parts: arm, ear, eye, finger, foot, hand, leg, lips, nose, toes

Clothing: coat, dress, hat, jacket, pants, shirt, shoe, skirt, sock, sweater
Fruit: apple, banana, cherry, grapes, lemon, orange, peach, pear, pineapple, strawberry

Furniture: bed, chair, couch, desk, dresser, fridge, rocking-chair, stool, table, TV

Musical instruments: accordion, bell, drum, flute, French horn, guitar, harp, piano, trumpet, violin

Tools: axe, chisel, hammer, paintbrush, pliers, ruler, saw, scissors, screwdriver, wrench

Vegetables: artichoke, asparagus, carrot, celery, lettuce, mushroom, onion, pepper, potato, pumpkin

Vehicles: airplane, bicycle, bus, car, helicopter, 780 motorbike, sailboat, train, truck, wagon 